\title{
Percepción de los Estudiantes Sobre la Utilización de Videojuegos en Cursos de la Universidad Nacional Abierta y a Distancia -Unad
}

\section{Students Perception on the Use of Video Games in Courses of THE Open and Distance National Univeritity - Unad}

\author{
'López Ortiz Iván Arturo, ${ }^{2}$ Hermes Mosquera Angulo \\ Universidad Nacional Abierta y a Distancia -UNAD, Popayán, Colombia \\ ${ }^{1}$ ivan.lope@unad.edu.co \\ ${ }^{2}$ hermes.mosquera@unad.edu.co
}

Recibido: 10/08/2015•Aprobado: 12/11/2015

\section{ResUMmen}

El artículo parte del contexto de la industria de los videos juegos en el ámbito mundial y la cantidad de dinero que esta actividad mueve alrededor del mundo (90.000 millones de dólares para el 2015 según [1]). También se describen algunas experiencias de éxito entorno a proyectos dedicados específicamente a la educación. Sin embargo, los resultados presentados acá son el punto de partida del proyecto de investigación denominado, Los video juegos como estrategia de enseñanza-aprendizaje en cursos de la Universidad Nacional Abierta y a Distancia -Unad- con el que se pretende dar respuesta a una de la preguntas orientadoras del proyecto: ¿Cuál es la percepción que tienen los estudiantes de educación superior sobre los video juegos? Y, en este sentido, la base fundamental de esta investigación es presentar un análisis de la aceptabilidad y uso del videojuego por parte de los estudiantes y profesores de diferentes programas de la Universidad Nacional Abierta y a Distancia, caracterizados por géneros, edades y programas académicos, entre otros aspectos. Como resultado, se obtuvo información importante sobre gustos, hábitos y dispositivos en los que los estudiantes tienen instalados los videojuegos, además, del tiempo que les dedican a estos, o estarían dispuestos a dedicarles en la semana. Con la información recolectada, ya es posible iniciar la construcción de un videojuego adecuado a las características propias de los estudiantes de la Universidad.

Palabras clave: cursos académicos, educación, gamificación, video juegos.

\section{Abstract}

This article starts from the context of the industry of video games at the global level and the amount of money that this activity moves around the world (90,000 million dollars in 2015 according to [1]). It also describes some experiences of success on projects devoted specifically to education. However, the results presented here are the starting point of the research Project called The video games as a teaching-learning strategy in courses of the Open and Distance National University - UNAD-, which 
aims to respond to one of the guiding questions of the project: What is the perception of higher education students, on the video games? In this sense, the fundamental basis of this research is to present an analysis of the acceptability and use of video games by students and teachers of different programs of UNAD, characterized by gender, age and academic programs, among other aspects. As a result, was obtained important information about likes, habits and devices in which the students have installed games; in addition, the time they devote or would be willing to spend in the week, to these games. With the information gathered, it is possible to start the construction of a videogame appropriate to the students characteristics, in the UNAD

Keywords: academic courses, education, gamification, video games.

\section{InTRODUCCIÓN}

La innovación constante en los procesos académicos y didácticos hace de la Universidad Nacional Abierta y a Distancia, Unad, una de las pioneras en el uso de las Tecnología de la Información y la Comunicación en la educación en Colombia; en este sentido, la presente propuesta enfoca su estudio al uso de los videos juegos educativos en las aulas, lo que [2] ha denominado game-based learning DGBL. Partiendo de investigaciones realizadas desde diversas perspectivas, con conclusiones y demostraciones reales de experiencias exitosas, se puede vislumbrar una amalgama de posibilidades tecnopedagógicas que, con el concurso de un enfoque multidisciplinar, permite el desarrollo de propuestas innovadoras que redunden en beneficio de la comunidad académica.

Desde este punto, el presente artículo parte de una revisión de lo que en cifras económicas mueve la industria del videojuego, las cuales para el año 2018 podrían superar 113.300 millones de dólares [1], además de relacionar algunas experiencias en las que los videojuegos aportan un papel muy importante en el proceso de formación académica. También se realiza la indagación de la percepción que los estudiantes tienen sobre los videojuegos, para lo cual se planteó como pregunta de investigación, para el proyecto: “¿Cómo involucrar videojuegos como estrategia de enseñanza aprendizaje en cursos de educación superior?", acompañada de las siguientes preguntas orientadoras:
¿Cuál es la percepción que tienen los estudiantes de educación superior sobre los videojuegos?

¿Los juegos diseñados para distintos programas y cursos de la educación superior cumplen con el propósito de desarrollar y fortalecer la capacidad cognitiva de los estudiantes para la construcción de nuevo conocimiento sobre una realidad específica?

El presente artículo trata de dar respuesta a la primera pregunta orientadora, referida a la percepción que los estudiantes de educación superior, y específicamente de la Unad, tienen sobre los videojuegos. Para dar respuesta a este interrogante, se planteó un objetivo específico: "Indagar acerca de la percepción que tienen los estudiantes de la Unad sobre los video Juegos".

\section{La Industria de los Videojuegos}

Haciendo un poco de historia, se tiene que los primeros videojuegos se desarrollaron a partir de la culminación de la Segunda Guerra Mundial, de la mano con los primeros momentos del nacimiento de la computación. No obstante, es en la década de los 80's en la que todos estos esfuerzos e investigaciones dan fruto con el nacimiento de Pcaman, Donkey Kong, Tetris o Mario Bros, cuyas marcas se han mantenido hasta la actualidad. Es así, entonces, como nace una industria que en la actualidad mueve más de 50 mil millones de dólares alrededor 
del mundo [1]. La industria logró su máximo apogeo de masificación con la aparición de los iPhone en el año 2007 y el surgimiento posterior de sistemas operativos móviles. Según [3], "El tamaño del mercado de los videojuegos para el año 2014 fue de unos 23.188 millones de euros con un crecimiento del $3 \%$ con respecto al año anterior".

En 2015, la industria de los videojuegos movió unos 90.000 millones de dólares y para 2018, podría superar los 113.300 millones, de acuerdo con datos de la firma [4] Unas cifras de escándalo que convierten, de hecho, al mercado de los videojuegos en la industria del entretenimiento y el ocio digital más rentable de todo el mundo, por encima de míticas categorías como el cine, las series o la lectura [1].

Para el caso de Colombia, [5] afirma: "El desarrollo de videojuegos en Colombia empezó en 2004 con una compañía llamada Inmersion Games", en efecto, la desarrolladora más grande del país.

Igualmente, en Colombia, las empresas más destacadas, aparte de Efecto Studios, son, TeraVision y Brainz, ubicadas en Bogotá, y Below The Game con sede en Bucaramanga. Estas cuatro empresas se han dedicado a desarrollar videojuegos de calidad y no se han concentrado en el público colombiano, aspecto que los ha llevado a obtener grandes contratos con firmas internacionales: Brainz tiene uno con Square Enix, empresa japonesa responsable de hacer juegos como Final Fantasy, y también con Gamevil, otra gigante desarrolladora en Corea del Sur. TeraVision trabaja con Disney, y Below the Game, desde hace poco, con Sony.

A pesar de la popularidad que tienen los videojuegos y de ser una de las industrias multimillonarias, surgen muchos interrogantes cuando se decide utilizarlos como una estrategia educativa. Entre ellas, las que se plantea [6] en su conferencia de Gamificación, en uno de sus apartes, "La Gamificación es una técnica de aprendizaje que traslada la mecánica de los juegos al ámbito educativo-profesional con el fin de conseguir mejores resultados, ya sea para absorber mejor algunos conocimientos, mejorar alguna habilidad, o bien recompensar acciones concretas, entre otros muchos objetivos" y en este sentido es importante mencionar a [7] donde se pectos a tener encuenta cunado se utiliza la gamificación en la Educación, entre las cuales se destacan: "cómo aplicar la gamificación, protocolos para diseñar o implementar la gamificación, plataformas de desarrollo, competencias que se pueden alcanzar con el desarrollo de los videojuegos", entre otros.

\section{EXPERIENCIAS DE ÉXITO}

Lo que nace en los años 80's como una experiencia totalmente aislada, en la actualidad se ha convertido en una línea de trabajo muy interesante. En la Tabla 1 se presentan algunas de las experiencias que se encuentran en marcha en la actualidad: 
TABLA I

EXPERIENCIAS EXITOSAS

\begin{tabular}{|c|l|l|l|}
\hline ÍTEM & \multicolumn{1}{|c|}{ NOMBRE } & \multicolumn{1}{|c|}{ DESCRIPCIÓN } & \multicolumn{1}{|c|}{ LINK } \\
\hline 1 & Kokori & $\begin{array}{l}\text { Juego de Biología Celular, } \\
\text { desarrollado en Chile. }\end{array}$ & http://www.kokori.cl/ \\
\hline 2 & MinecraftEDU & $\begin{array}{l}\text { Versión del popular Minecraf dedicado } \\
\text { a enseñar contenidos educativos. }\end{array}$ & http://education.minecraft.net/ \\
\hline 3 & SimCityEDU & $\begin{array}{l}\text { Juego dedicado principalmente a } \\
\text { aspectos a gobernar una ciudad por } \\
\text { parte de los participantes del juego }\end{array}$ & http://www.glasslabgames.org/games/SC \\
\hline 4 & Hakitzu & $\begin{array}{l}\text { Juego de lucha para ganar batallas en- } \\
\text { tre robots, para aprender a programar. }\end{array}$ & $\begin{array}{l}\text { https://itunes.apple.com/es/app/hakitzu-elite-robot- } \\
\text { hackers/id599976903?mt=8 }\end{array}$ \\
\hline 5 & Classcraft & $\begin{array}{l}\text { Personalización de clases } \\
\text { mediante un juego de roles. }\end{array}$ & http://www.classcraft.com/es/ \\
\hline 6 & Dragon Box: & $\begin{array}{l}\text { Videojuego para enseñanza } \\
\text { de álgebra. }\end{array}$ & http://dragonbox.com/ \\
\hline 7 & Cap Odyssey & $\begin{array}{l}\text { Enseñanza de los principios } \\
\text { de la economía agrícola. }\end{array}$ & http://www.capodyssey.eu/ \\
\hline 8 & Simple Machines & Juego para diseño de máquinas. & \\
\hline
\end{tabular}

\section{DesarRollo de la Investigación}

El proceso investigativo enfoca su estudio al uso de los video juegos educativos (game-based learning) como estrategia de enseñanza aprendizaje al interior de los cursos de la Universidad. Partiendo de la revisión bibliográfica, con análisis de investigaciones realizadas desde diversas perspectivas, con base en conclusiones y demostraciones reales de experiencias exitosas, se puede vislumbrar una amalgama de posibilidades tecno-pedagógicas que, con el concurso de un enfoque multidisciplinar, permite el desarrollo de propuestas innovadoras que, sin lugar a dudas, pueden redundar en beneficio de la comunidad académica.

Desde este punto de vista, el proyecto parte de la indagación de la percepción que los estudiantes tienen sobre los videojuegos respondiendo a la pregunta orientadora formulada en el proyecto: “¿Cuál es la percepción que tienen los estudiantes de educación superior sobre los videojuegos?" y para lo cual se planteó el objetivo específico "Indagar sobre la percepción que tienen los estudiantes de la Unad sobre los videojuegos".

Por tanto y de acuerdo con la recopilación, clasificación y análisis de los datos obtenidos en dicha indagación, se podrá realizar un estudio para proponer una estrategia innovadora en los cursos académicos de la Unad, como Institución Universitaria que basa su campo de acción en su misión institucional que en algunos de sus apartes menciona: "fomentar y acompañar el aprendizaje autónomo para contribuir a la educación para todos a través de la modalidad abierta, a distancia y en ambientes virtuales de aprendizaje, con la utilización de las tecnologías de la información y la comunicación" [8].

\section{A. Alcance metodológico}

La presente investigación tiene un alcance descriptivo de diseño no experimental, de corte 
transversal con un enfoque meramente cuantitativo, que a través de mediciones numéricas busca cuantificar, reportar e indagar acerca de la percepción que los estudiantes de la Unad tienen sobre los videojuegos. Por tanto y con el fin de interpretar la información recopilada, se utiliza la estadística, presentando los resultados por medio de gráficas que aportan potencialmente mayor valor de análisis cuantitativo. Este enfoque es un proceso que recolecta, analiza y vincula datos cuantitativos en la investigación para responder a la pregunta antes planteada, aplicable para cualquier campo de la investigación de las Ciencias Físico-Naturales. Se utiliza la encuesta como instrumento para recoger los datos, los cuales también incluyen la medición sistemática, y su análisis como característica relevante [9].

\section{B. Estudio metodológico}

El estudio metodológico se realizó en tres fases:

La Fase 1, correspondiente a la fase inicial de la investigación, consistió en la exploración y revisión bibliográfica acerca de los videojuegos como recurso educativo; se acompañó esta fase, con la observación directa de fuentes externas, como medio para familiarizarse con el tema. Esto permitió un acercamiento a la temática de los videojuegos, sus posibilidades, alcances y oportunidades de innovación y mejoramiento continuo.

En la Fase 2 se realizó el diseño y la aplicación del instrumento encuesta como medio para recolección de la información; seguido a este, se llevó a cabo el procesamiento, análisis e interpretación de los resultados obtenidos de la Fase 1.

En la Fase 3 se planteó el diseño e implementación de un videojuego, aplicado a un curso académico de la Universidad, tendiente a conocer el impacto que este tendría en el proceso formativo, así como, las conclusiones y recomendaciones que servirán como insumo para la innovación de los cursos académicos al interior de la Universidad.

\section{Población y muestra}

La muestra fue aleatoria, conformada por 407 estudiantes de un total aproximado de 60.000 , ubicados en diferentes zonas geográficas del país y matriculados en diversos programas académicos ofertados por la Universidad. El margen de error fue del $5 \%$, con un nivel de confianza del $96 \%$.

\section{Técnica de recolección de información}

Se utilizó la encuesta como instrumento de recolección de información. Consistió en un cuestionario aplicado en línea, previamente elaborado y validado por la red académica y el equipo investigador, valorando aspectos como: univocidad (muy claro, resulta comprensible y presenta ambigüedades), pertinencia (muy pertinente, pertinente y poco pertinente) e importancia (muy importante, algo importante y poco importante); estos criterios se aplicaron tanto al cuestionario como al encabezado mismo, lo que permitió realizar varios ajustes y versiones al instrumento.

\section{E. Análisis e interpretación de los resultados}

A continuación, se presenta el análisis y la interpretación de los datos que arrojó la encuesta. Teniendo en cuenta la selección diversa de la muestra, se logró obtener una mirada global hacia la percepción de los videojuegos como recurso educativo.

Tiempo promedio que utiliza el computador en la semana Fig. 1.

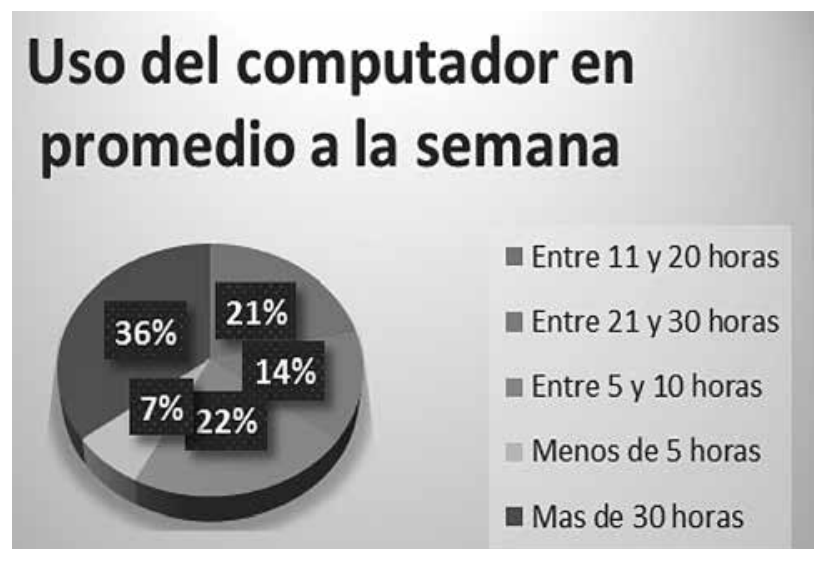

Fig. 1 Resultado del uso de computadora promedio por semana. 
De los datos anteriores, se pudo establecer que los estudiantes de la Unad hacen uso de la computadora en un promedio de $\mathbf{2 0}$ horas a la semana, lo cual está por encima del promedio nacional que es de 14,35 horas de acuerdo con datos de [10] en su informe anual de tendencias, aclarando que solo se está haciendo el compartivo con uso de computador y no de otro dispositvo como Smartphone.

\section{- De los siguientes dispositivos, en cuál usted tiene instalados videojuegos según Tabla 2 y Fig. 2:}

TABLA II

Análisis Dispositivos con Video Juegos

\begin{tabular}{|c|c|c|}
\hline DISPOSITIVO & CANTIDAD & ÍNDICE \\
\hline Computador & 51 & $11,0 \%$ \\
\hline Computador, consola. & 9 & $1,9 \%$ \\
\hline Computador, móvil - celular. & 78 & $16,8 \%$ \\
\hline $\begin{array}{l}\text { Computador, móvil - celular, } \\
\text { consola. }\end{array}$ & 22 & $4,8 \%$ \\
\hline $\begin{array}{l}\text { Computador, móvil - celular, } \\
\text { móvil - tablet. }\end{array}$ & 31 & $6,7 \%$ \\
\hline $\begin{array}{l}\text { Computador, móvil - celular, } \\
\text { móvil - tablet, consola. }\end{array}$ & 29 & $6,3 \%$ \\
\hline Computador, móvil - tablet. & 7 & $1,5 \%$ \\
\hline $\begin{array}{l}\text { Computador, móvil - } \\
\text { tablet, consola. }\end{array}$ & 3 & $0,6 \%$ \\
\hline Consola & 10 & $2,2 \%$ \\
\hline Móvil - celular. & 70 & $15,1 \%$ \\
\hline Móvil - celular, consola. & 7 & $1,5 \%$ \\
\hline Móvil - celular, móvil - tablet. & 19 & $4,1 \%$ \\
\hline $\begin{array}{l}\text { Móvil - celular, móvil - tablet, } \\
\text { consola. }\end{array}$ & 4 & $0,9 \%$ \\
\hline $\begin{array}{l}\text { Móvil - celular, no tengo jue- } \\
\text { gos instalados. }\end{array}$ & 2 & $0,4 \%$ \\
\hline Móvil - tablet. & 13 & $2,8 \%$ \\
\hline Móvil - tablet, consola. & 1 & $0,2 \%$ \\
\hline $\begin{array}{l}\text { No tengo juegos } \\
\text { instalados. }\end{array}$ & 107 & $23,1 \%$ \\
\hline
\end{tabular}

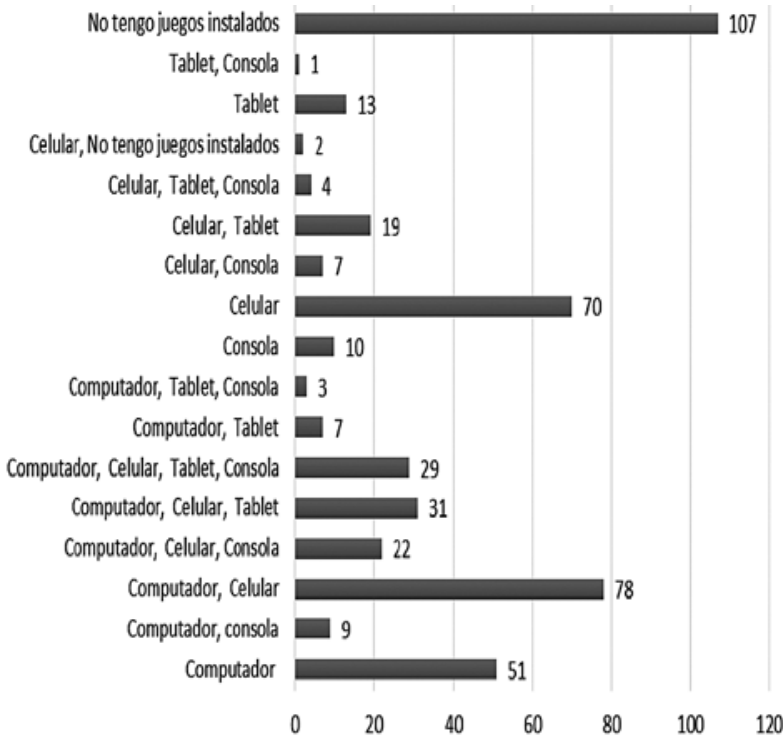

Fig. 2 Resultado tendencia de uso de dispositivos con videojuegos

En esta pregunta resulta interesante observar que un buen número de los estudiantes no tiene instalado videojuegos; sin embargo, el $77 \%$ de los encuestados afirma que sí los tiene instalados (Fig. 3) en alguno de los dispositivos, siendo la tendencia a instalarlos tanto en las computadoras como en sus celulares, lo que está en concordancia con el estudio publicado por [11], en el que se indica que el computador alcanza el $56.3 \%$ y los móviles el 52,2\%; sin embargo y con este mismo estudio, la tendencia puede cambiar hacia el uso de consolas y dispositivos móviles.

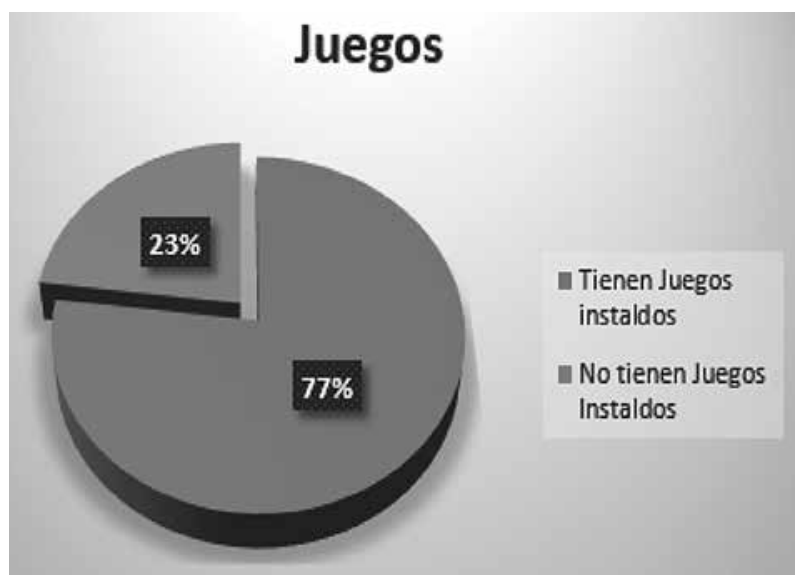

Fig. 3 Resultado de usuarios con videojuegos 
- Valoración general de los videojuegos. Tabla 3 y Fig. 4.

TABLA III

Valoración General de Videojuegos

\begin{tabular}{|l|c|c|}
\hline CALIFICACIÓN & CANTIDAD & ÍNDICE \\
\hline Buenos & 236 & $51,0 \%$ \\
\hline Muy buenos & 122 & $26,3 \%$ \\
\hline Malos & 13 & $2,8 \%$ \\
\hline Muy malos & 1 & $0,2 \%$ \\
\hline Regulares & 91 & $19,7 \%$ \\
\hline
\end{tabular}

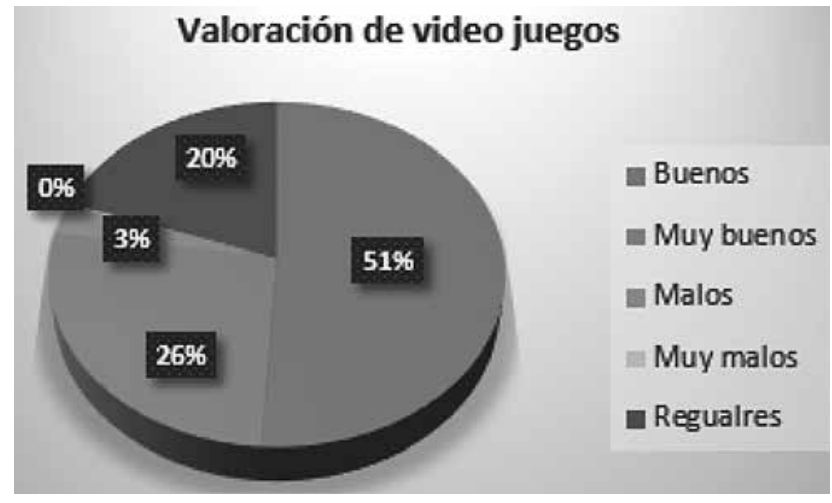

Fig. 4 Resultado valoración de videojuegos

Se observa, como una valoración general de los videojuegos, que la mayoría de los estudiantes de la universidad considera que son buenos.

\section{- Percepción del uso de videojuegos por sexo.} Según Tabla 4 y Fig. 5.

TABLA IV

Percepción de Uso de Videojuegos

\begin{tabular}{|l|c|c|}
\hline INTENSIDAD DE USO & CANTIDAD & ÍNDICE \\
\hline Hombres & 455 & $98,3 \%$ \\
\hline Mujeres & 8 & $1,7 \%$ \\
\hline
\end{tabular}

\section{Percepción de mayor uso} de videojuegos

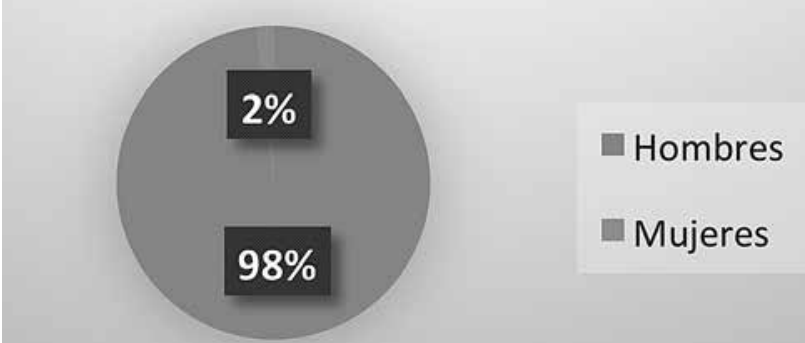

Fig. 5 Resultado percepción de uso de videojuegos

Los resultados obtenidos acerca de la percepción de mayor uso de videojuegos entre géneros indican que los encuestados piensan que los hombres los usan más; sin embargo y al contrastar con cada interrogante, se puede ver que tanto hombres como mujeres los utilizan, más aun, cuando el $40 \%$ de los estudiantes encuestados son mujeres. Esto se puede corroborar también con el estudio de [12], en el que se encuentra que los hombres solo superan a las mujeres en un 10\%: "Esta estadística presenta la distribución de los jugadores de videojuegos en España durante el cuarto trimestre de 2014, por edad y género. Los hombres, con un porcentaje del $54 \%$, superaron a las mujeres, con un $46 \%$. "

- Cantidad de horas promedio que dedican o podrían dedicar a los videojuegos. Tabla 6 y Fig. 5.

TABLA VI

Tiempo Dedicado a los Videos

\begin{tabular}{|l|c|c|}
\hline INTENSIDAD DE USO & CANTIDAD & ÍNDICE \\
\hline Cero horas. & 122 & $26,3 \%$ \\
\hline Entre 1 a 5 horas. & 290 & $62,6 \%$ \\
\hline Entre 6 a 10 horas. & 50 & $10,8 \%$ \\
\hline Entre 11 y 20 horas. & 17 & $3,7 \%$ \\
\hline Entre 21 y 30 horas. & 1 & $0,2 \%$ \\
\hline Más de 30 horas. & 3 & $0,6 \%$ \\
\hline
\end{tabular}




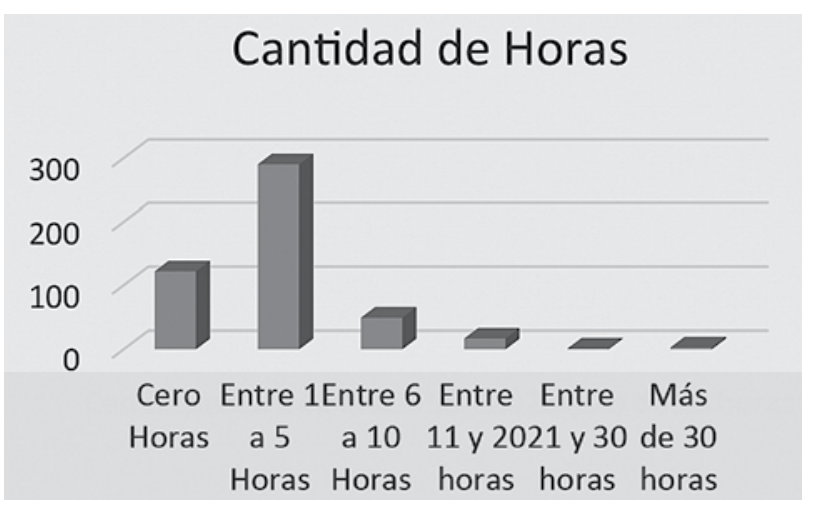

Fig. 5 Resultado tiempo dedicado a los video juegos

Los datos arrojaron que el $60 \%$ de los encuestados dedica a los videojuegos entre 1 y 5 horas al día, muy por debajo del tiempo que los adolescentes pueden pasar jugando, el cual oscila entre 9 horas al día según estudio publicado por [13]

- ¿Si tiene oportunidad de jugar, permanece todo el tiempo en el mismo juego? Tabla $7 \mathrm{y}$ Fig. 6.

TABLA VII

Permanencia en el Juego

\begin{tabular}{|l|c|c|}
\hline CRITERIO & CANTIDAD & ÍNDICE \\
\hline Sí & 172 & $37,1 \%$ \\
\hline No & 206 & $44,5 \%$ \\
\hline No juego. & 85 & $18,4 \%$ \\
\hline
\end{tabular}

\section{Permanencia en el mismo juego}

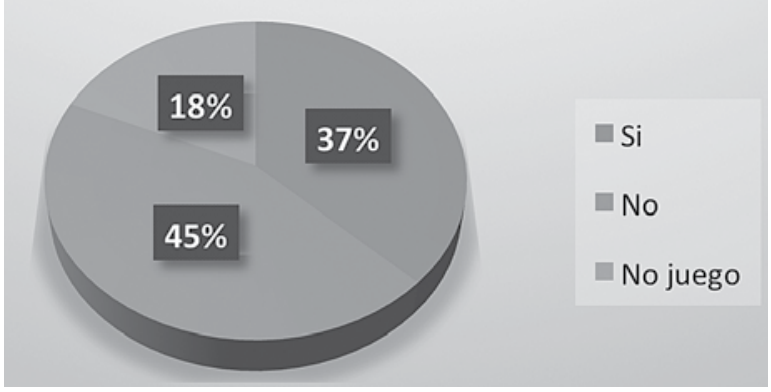

Fig. 6 Resultado permanencia en el juego
El comportamiento para los estudiantes de la Universidad en cuanto a la permanencia, indica que el $44 \%$ prefiere cambiar de juego con alguna frecuencia.

\section{- ¿Prefiere jugar acompañado o solo?} Tabla 8 y Fig. 7.

TABLA VIII

Preferencia por Jugar

\begin{tabular}{|l|c|c|}
\hline CRITERIO & CANTIDAD & ÍNDICE \\
\hline Acompañado & 228 & $49,2 \%$ \\
\hline Solo & 235 & $50,8 \%$ \\
\hline
\end{tabular}

\section{Percepción de compañía cuando juega}

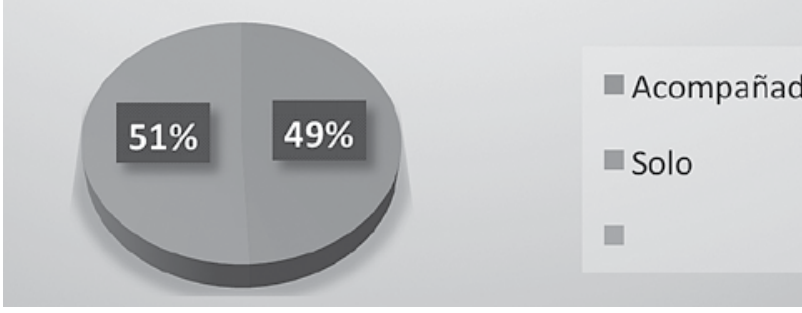

Fig. 7 Resultado preferencia por jugar

En relación con la preferencia de los encuestados de estar solos o acompañados al momento de jugar, se encontró que el $49 \%$ prefiere estar acompañado, mientras que el $51 \%$ prefiere estar solo, lo que es interesante, dado que otros estudios, como el de [14] permite determinar que en Brasil también las personas que juegan prefieren hacerlo solas (42\%); sin embargo, un alto porcentaje afirma que es más divertido jugar acompañado.

- Tipos de juegos que más le llaman la atención según Tabla 9. 
TABLA IX

TiPOS DE JUEgos

\begin{tabular}{|c|c|c|}
\hline TIPOS DE JUEGOS & CANTIDAD & ÍNDICE \\
\hline $\begin{array}{l}\text { Juegos de estrategia } \\
\text { (aventuras gráficas, rol } \\
\text { y guerra). }\end{array}$ & 111 & $24,0 \%$ \\
\hline $\begin{array}{l}\text { Juegos de simulación } \\
\text { (instrumentales, situacionales } \\
\text { y deportivos). }\end{array}$ & 75 & $16,2 \%$ \\
\hline $\begin{array}{l}\text { Juegos de simulación } \\
\text { (instrumentales, situacionales } \\
\text { y deportivos). Juegos de } \\
\text { estrategia (aventuras gráficas, } \\
\text { rol y guerra). }\end{array}$ & 31 & $6,7 \%$ \\
\hline $\begin{array}{l}\text { Jugo de arcade (plataformas, } \\
\text { laberintos, deportivos, dispara } \\
\text { y olvida). }\end{array}$ & 94 & $20,3 \%$ \\
\hline $\begin{array}{l}\text { Jugo de arcade (plataformas, } \\
\text { laberintos, deportivos, dispara } \\
\text { y olvida). Juegos de estrate- } \\
\text { gia (aventuras gráficas, rol y } \\
\text { guerra). }\end{array}$ & 52 & $11,2 \%$ \\
\hline $\begin{array}{l}\text { Jugo de arcade (plataformas, } \\
\text { laberintos, deportivos, dispara } \\
\text { y olvida). Juegos de simula- } \\
\text { ción (instrumentales, situacio- } \\
\text { nales y deportivos). }\end{array}$ & 23 & $5,0 \%$ \\
\hline $\begin{array}{l}\text { Jugo de arcade (plataformas, } \\
\text { laberintos, deportivos, dispara } \\
\text { y olvida). Juegos de simula- } \\
\text { ción (instrumentales, situacio- } \\
\text { nales y deportivos). Juegos de } \\
\text { estrategia (aventuras). }\end{array}$ & 40 & $8,6 \%$ \\
\hline
\end{tabular}

Se puede apreciar que a los estudiantes de la Universidad les interesan mucho los juegos de estrategias (aventuras gráficas, rol y guerra).

\section{- Temáticas que prefieren en los videojuegos.} Tabla 10 y Fig. 8.
TABLA X

Temáticas en los Videojuegos

\begin{tabular}{|l|c|c|}
\hline TEMÁTICAS & CANTIDAD & ÍNDICE \\
\hline $\begin{array}{l}\text { De violencia, deportivos, } \\
\text { educativos, temas generales. }\end{array}$ & 71 & $15,3 \%$ \\
\hline $\begin{array}{l}\text { Deportivos, educativos, temas } \\
\text { generales. }\end{array}$ & 130 & $28,1 \%$ \\
\hline Educativos, temas generales. & 133 & $28,7 \%$ \\
\hline Temas generales. & 112 & $24,2 \%$ \\
\hline
\end{tabular}

\section{Temáticas de preferencia en los juegos}

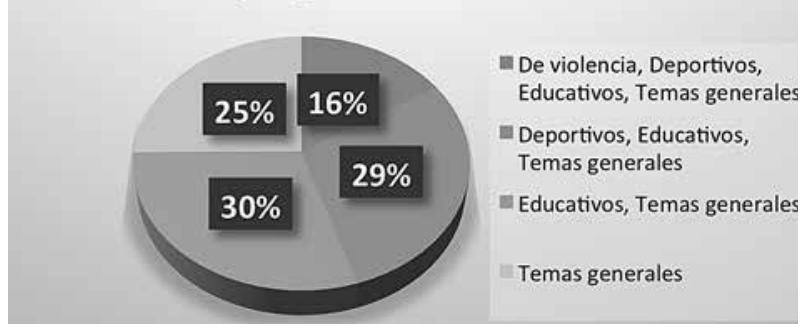

Fig. 8 Resultado tipo de juegos que le llaman la atencón.

Contrastando este ítem con el anterior, es interesante observar, que al dirigir la pregunta de la consulta de forma más específica, se encuentra una mayor tendencia hacia los juegos educativos.

Es así, como los resultados arrojan que el 30\% prefiere temas educativos y generales, mientras que el $16 \%$ tiene preferencia por los juegos de temáticas de violencia o acción. En este sentido, los estudiantes de la Unad no están inclinados con los juegos de acción como lo muestra el estudio de [15] (dirigido a los españoles), en el que el $62,7 \%$ se inclina por estos últimos.

- Habilidades que desarrolla el videojuego. Tabla 11 y Fig. 9. 
TABLA XI

Habilidades en los Videojuegos

\begin{tabular}{|l|c|c|}
\hline HABILIDADES & CANTIDAD & ÍNDICE \\
\hline $\begin{array}{l}\text { Analíticas, toma de decisiones, } \\
\text { resolución de problemas. }\end{array}$ & 9 & $1,9 \%$ \\
\hline $\begin{array}{l}\text { Asimilación y retención } \\
\text { de la información. }\end{array}$ & 36 & $7,8 \%$ \\
\hline $\begin{array}{l}\text { Atención, asimilación y } \\
\text { retención de la información. }\end{array}$ & 117 & $25,3 \%$ \\
\hline $\begin{array}{l}\text { Creativas, analíticas, metacog- } \\
\text { nitivas (aprender a aprender). }\end{array}$ & 22 & $4,8 \%$ \\
\hline $\begin{array}{l}\text { Metacognitivas (aprender } \\
\text { a aprender). }\end{array}$ & 10 & $2,2 \%$ \\
\hline $\begin{array}{l}\text { Para la búsqueda } \\
\text { de la información. }\end{array}$ & 6 & $1,3 \%$ \\
\hline $\begin{array}{l}\text { Psicomotrices, atención, } \\
\text { creativas. }\end{array}$ & 245 & $52,9 \%$ \\
\hline Resolución de problemas. & 6 & $1,3 \%$ \\
\hline \begin{tabular}{l} 
Toma de decisiones. \\
\hline
\end{tabular} & $8 \%$ \\
\hline
\end{tabular}

Percepción de habilidades que posibilitan los juegos

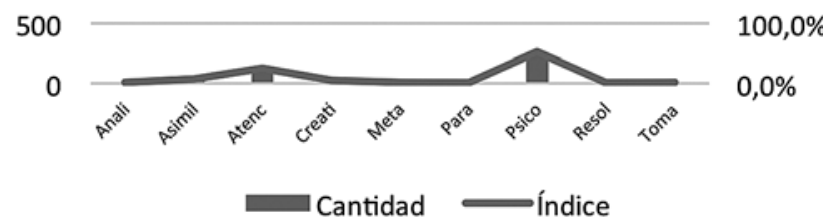

Fig. 9 Resultado habilidades que desarrollan los videojuegos.

Al indagar sobre la opinión que tienen los encuestados acerca de las habilidades que desarrollan los videojuegos, el 52,9\% considera que desarrolla habilidades psicomotrices, de atención y creatividad, lo que está muy ligado al estudio publicado en [16], en el que se encuestaron a 2.876 personas de todas las edades y se encontró que: "Además los videojuegos potenciarían habilidades como el liderazgo, ya que más de la mitad de los menores de 35 años encuestados afirmaron haberlo potenciado, y por otro lado la capacidad de superación: un 53,62 por ciento aseguró que los videojuegos han influido mucho en la mejora de esta característica."
- ¿Para qué considera que sirven los videojuegos? Tabla 12 y Fig. 10.

TABLA XII

Utilidad de los Videojuegos

\begin{tabular}{|l|c|c|}
\hline UTILIDAD DE LOS JUEGOS & CANTIDAD & ÍNDICE \\
\hline $\begin{array}{l}\text { Ayudar a relacionarse con otras perso- } \\
\text { nas, pasar el tiempo, ejercicio mental. }\end{array}$ & 20 & $4,3 \%$ \\
\hline Conseguir objetivos educativos. & 39 & $8,4 \%$ \\
\hline Desestresarse & 3 & $0,6 \%$ \\
\hline Ejercicios mentales. & 3 & $0,6 \%$ \\
\hline Mejora la auto estima. & 3 & $0,6 \%$ \\
\hline Mejorar el aprendizaje. & 32 & $6,9 \%$ \\
\hline $\begin{array}{l}\text { Motivar, alcanzar objetivos educativos, } \\
\text { pasar el tiempo, mejorar el aprendizaje. }\end{array}$ & 245 & $52,9 \%$ \\
\hline Pasar el tiempo. & 117 & $25,3 \%$ \\
\hline
\end{tabular}

Percepción de la utilidad de los juegos

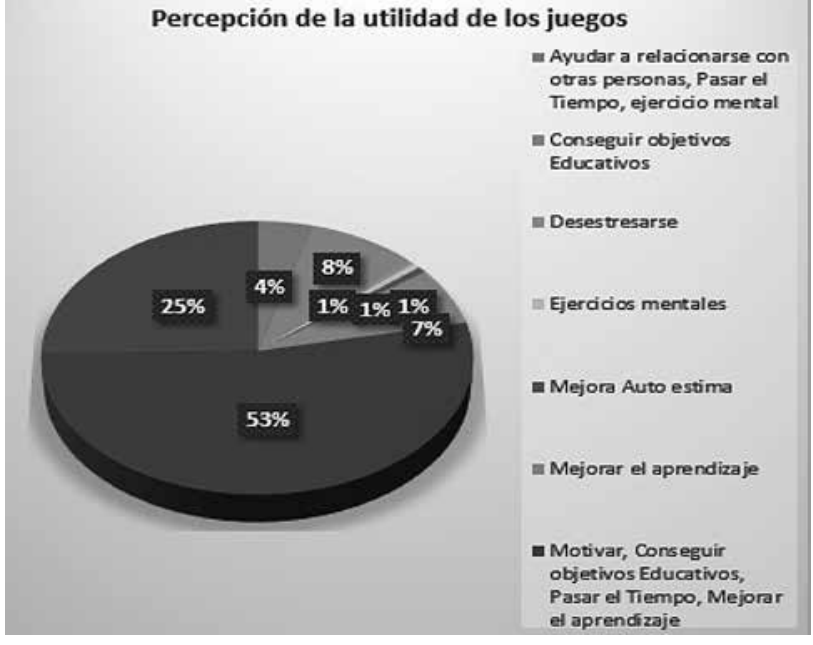

Fig. 10 Resultado utilidad de los Videojuegos

De acuerdo con la percepción de los encuestados frente a la utilidad de los videojuegos, se encontró que el $53 \%$ piensa que sirven para motivar y alcanzar objetivos educativos. En este sentido y aprovechando esta percepción de los estudiantes sobre este punto específicamente, se pueden potenciar los cursos de la Unad.

\section{- ¿Por qué son atractivos los videojuegos? Tabla 13 y Fig. 11.}


TABLA XIII

Atractivo de los Videojuegos

\begin{tabular}{|l|c|c|}
\hline PERCEPCIÓN & CANTIDAD & ÍNDICE \\
\hline Permiten analizar y pensar. & 1 & $0,2 \%$ \\
\hline Diversión & 1 & $0,2 \%$ \\
\hline Vicio & 1 & $0,2 \%$ \\
\hline \begin{tabular}{l} 
Entretienen y enseñan. \\
\hline $\begin{array}{l}\text { Es una forma visual y } \\
\text { didáctica de aprendizaje. }\end{array}$
\end{tabular} & 1 & $0,2 \%$ \\
\hline $\begin{array}{l}\text { Existe una meta } \\
\text { que se debe alcanzar. }\end{array}$ & 400 & $8,2 \%$ \\
\hline $\begin{array}{l}\text { Permiten un recuento } \\
\text { automático de puntos. }\end{array}$ & 8 & $1,7 \%$ \\
\hline $\begin{array}{l}\text { Por el azar. } \\
\text { Tienen imágenes visuales } \\
\text { en movimiento. }\end{array}$ & 44 & $0,6 \%$ \\
\hline
\end{tabular}

\section{Cantidad}

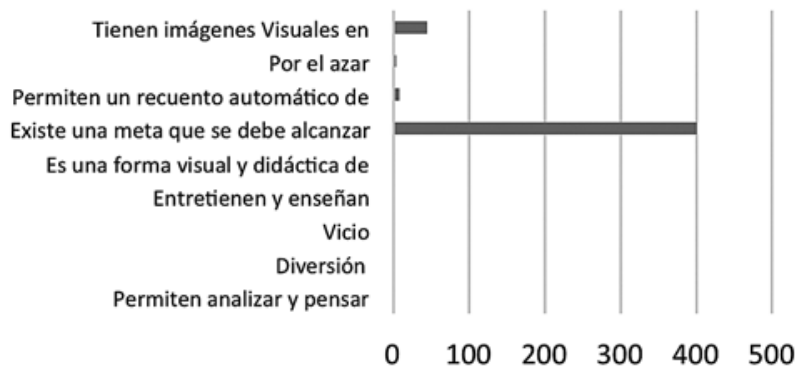

Fig. 11 Resultado de lo atractivo de los videojuegos

El $85 \%$ de los estudiantes de la Unad, considera que lo atractivo de los juegos se centra en que existe una meta por alcanzar. Esto puede tener una explicación interesante, de acuerdo con lo planteado por [17], psicólogo e investigador de la cultura digital, quien manifiesta que "la mayor o menor adicción que provoca un videojuego también tiene que ver con cómo escalona las recompensas y las correspondientes frustraciones. Los juegos no atrapan por diversión, sino por su estructura, porque te plantean hacer determinadas cosas que son asequibles pero que a la vez entrañan ciertas complicaciones, y cada vez que fallas o ves que puedes mejorar y hacerlo más rápido se crea un desequilibrio que buscas equilibrar rápidamente, jugando de nuevo."
- ¿Los cursos de la Unad deberían introducir videojuegos en sus temáticas? Tabla $14 \mathrm{y}$

Fig. 12.

TABLA XIV

Inclusión de Videojuegos en Cursos

\begin{tabular}{|l|c|c|}
\hline DECISIÓN & CANTIDAD & ÍNDICE \\
\hline Sí & 401 & $86,6 \%$ \\
\hline No & 62 & $13,4 \%$ \\
\hline
\end{tabular}

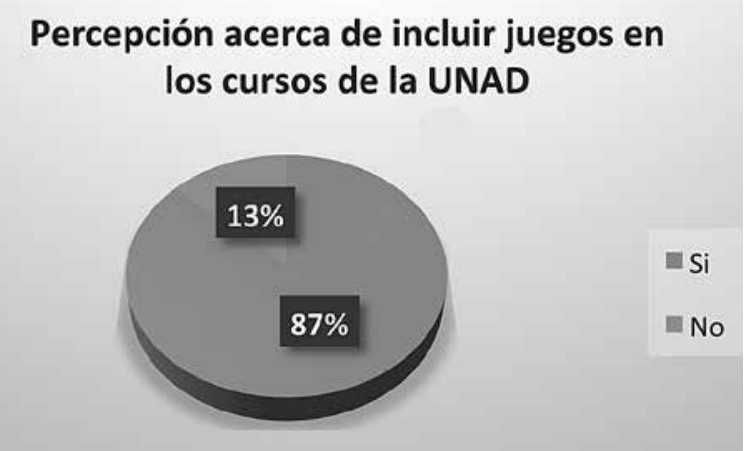

Fig. 12 Resultado inclusión de videojuegos en cursos de la Unad

Al consultar a los estudiantes sobre el incluir videojuegos en los cursos de la Universidad, el resultado fue arrollador: un $87 \%$ está a favor de que se considere esta posibilidad.

\section{Conclusiones y Trabajo Futuro}

De acuerdo con los resultados obtenidos en la encuesta, se puede concluir que los estudiantes de la Unad:

- Utilizan el computador en un promedio por arriba de la tasa media nacional.

- Sí tienen instalados videojuegos en alguno de sus dispositivos tecnológicos.

- Consideran en términos generales que los videojuegos son buenos, sin una especificación de tipo de juego, da la sensación que para los estudiantes de la universidad asumen los juegos desde el contexto educativos 
- Tienen una percepción errada en el sentido de que los hombres son los que hacen mayor uso de los videojuegos.

- Tienen una alta posibilidad de engancharse con videojuegos, dedicando más de una hora al día a estos.

- No les llama mucho la atención permanecer tanto tiempo en el mismo juego, lo que con lleva a pensar en una buena estrategia de motivación, en el caso de que se implementen juegos para los cursos de la Universidad.

- Muy por debajo de lo que se esperaba, les gusta jugar de manera acompañada o interactuar con los juegos.

- En relación con los tipos de juegos que les llama la atención, les gusta los juegos de acción y los educativos, lo que permite idear juegos de acción educativos.

- Perciben que los juegos los motivan a superar retos.

- Están muy de acuerdo en que los videojuegos sean incluidos en los cursos.

Con base en estos resultados, es importante para el grupo elaborar ya la estrategia de la construcción de un video juego que cumpla con muchas de las características mencionadas por los usuarios.

\section{ReCOMENDACIONES}

De acuerdo a los datos obtenidos se recomienda a los funcionarios que trabajan en el diseño tecnoedagogico de la universidad, dar una mirada a este tipo de recurso y proponer dar las bases para la construcción o utilización de los videojuegos en los cursos académicos.

Según lo indagado en las encuestas, los estudiantes de la universidad le llaman la atención que en sus cursos existirá este tipo de recursos y en este sentido es importante continuar con este tipo de proyectos para tener productos propios y mostrables a la comunidad académica.

El trabajo de diseño y construcción de un video juego involucra muchos actores: diseñadores, programadores y productores de sonido, entre otros, con el fin de elaborar productos de buena calidad, a la altura de instituciones como la Unad.

Invitar a la comunidad académica a diseñar y utilizar recursos como los juegos como estrategia innovadora que motiven a los estudiantes a la continuidad en la universidad

\section{RefERENCIAS}

[1] A. Iglesias, «ticbeat,» 6 Febrero 2016. [En línea]. Available: http://www.ticbeat.com/cyborgcultura/videojuegos-la-industria-multimillonaria-detras-del-mayor-entretenimiento-delplaneta/. [Último acceso: 30 Mayo 2016].

[2] M. Prensky, «ACM,» 1 Octubre 2003. [En línea]. Available: http://dl.acm.org/citation.cfm?id=950596. [Último acceso: 20 Noviembre 2015].

[3] EAE Busuness Scholl, «eae,» 2014. [En línea]. Available: http://www.eae.es/actualidad/noticias/el-mercado-del-videojuego-en-espana-movio-763-millones-de-en-2014-conun-crecimiento-del-31-respecto-al-2013.

[4] newzoo, «newzoo,» 2015. [En línea]. Available: https://newzoo.com/insights/trend-reports/.

[5] C. Echavarria, «vice,» 23 Julio 2015. [En línea]. Available: http://www.vice.com/es_co/read/videojuegos-made-in-colombia. [Último acceso: Mayo 2016].

[6] E. Garcia, «CongresoElearning,» 18 Octubre 2015. [En línea]. Available: http://www.congresoelearning.org/profiles/ blogs/preguntas-conferencia-gamificaci-n-y-educaci-n-1. [Último acceso: 23 Mayo 2016].

[7] V. Gaitán, «Educativa,» 15 Octubre 2013. [En línea]. Available: http://www.educativa.com/blog-articulos/gamificacion-elaprendizaje-divertido/. [Último acceso: 12 Novimebre 2015].

[8] UNAD, «UNAD,» 12 Junio 2014. [En línea]. Available: http:// informacion.unad.edu.co/acerca-de-la-unad/mision-y-vision. [Último acceso: 30 Mayo 2016].

[9] R. Hernández, Metodología de la Invetigación, Mexico: McGraw Hill, 2004.

[10] M. Meeker, «KPCB,» Enro 2016. [En línea]. Available: http://www.kpcb.com/internet-trends?utm_content= buffer14592\&utm_medium=social\&utm_source=twitter. com\&utm_campaign=buffer. 
[11] Economía Digital, «Expansion,» 2016. [En línea]. Available: http://www.expansion.com/economia-digital/innovacion/201 6/01/28/56aa4fd3ca4741e82e8b463c.html.

[12] Statista, «statista,» 2014. [En línea]. Available: https:// es.statista.com/estadisticas/481369/jugadores-de-videojuegos-en-espana-por-edad-y-genero/.

[13] CNN, «cnnespanol,» 2015. [En línea]. Available: http://cnnespanol.cnn.com/2015/11/03/los-adolescentes-pasan9-horas-al-dia-usando-los-medios-segun-informe/.

[14] Fundación Telefonica, «Adiccionvideojuegos,» 2014. [En línea]. Available: https://adiccionvideojuegos.wordpress.com/ tag/estadisticas/.
[15] GrupAd Hoc, «slideplayer,» 2015. [En línea]. Available: http://slideplayer.es/slide/1436871/.

[16] Edicicones el País, «tecnología el país,» 2013. [En línea]. Available: http://tecnologia.elpais.com/tecnologia/2005/12/22/actualidad/1135243680_850215.html.

[17] R. Balaguer, «lavanguradia,» 2013. [En línea]. Available: http://www.lavanguardia.com/estilos-de-vida/20120629/ 54317381414/por-que-enganchan-los-videojuegos.html. 
